\title{
Cosmography with high-redshift probes
}

\author{
Vincenzo Vitagliano \\ CENTRA, Instituto Superior Técnico, \\ Universidade de Lisboa, Av. Rovisco Pais 1, 1049 Lisboa, Portugal \\ email: vincenzo.vitagliano@ist.utl.pt
}

\begin{abstract}
I discuss how the cosmographic approach to the determination of cosmological parameters can be implemented with the inclusion of high-redshift data. I argue on the viability of such high- $z$ probes for cosmographic purposes, and resume some statistical issues in finding the most reliable cosmographic truncation.
\end{abstract}

Keywords. cosmological parameters, cosmology: miscellaneous

\section{Introduction}

Cosmology has recently become one of the most severe referee testing the reliability of theories alternative to General Relativity and models beyond standard particle physics. The analysis of cosmological data and the extraction of consistent results require the definition of a specific underlying theoretical model. The next natural question is then whether or not it is possible to define a scheme with minimal, self-consistent dynamical requests allowing the analysis of cosmological observations while skipping the bias of any theoretical prior.

Cosmography provides one possibility of such tool: starting with the educated guess of a cosmological metric described by the spatially homogeneous and isotropic FriedmannLemaitre-Robertson-Walker solution, any relevant distance indicator of an observed object can be then expanded in a power series of a suitable redshift parameter. The coefficients of such powers (evaluated today), casted into a combination of successive weighted derivatives of the scale factor $a(t)$ (the cosmographic parameters), contain the relevant information for a kinematic description of the universe.

It has to be stressed that the ill-behaviour at high redshift of the expansion is known to strongly affect the results. To circumvent the problem one must abandon the standard relation linking the luminosity distance to the ordinary-defined redshift. As already pointed out in Cattoen \& Visser (2007), the lack of validity of the Taylor-expanded expression for the luminosity distance could be settled down approximately at $z \sim 1$. In order to avoid problems with the convergence of the series for the highest redshift objects as well as to control properly the approximation induced by truncations of the expansions and the underestimation of the errors, it is useful to recast the luminosity distance as a function of an improved parameter $y=z /(1+z)$ (Visser (2004), Cattoen \& Visser (2007)). In such a way, being $z \in(0, \infty)$ mapped into $y \in(0,1)$, one is in principle able to retrieve the right behaviour for series convergence at any distance. The introduction of this new redshift variable will not affect the definition of the cosmographic parameters.

\section{The data ensemble}

Reaching the highest possible redshift allowed by data is a fundamental condition to disentangle between competing cosmological models. Given that most of the models are 
built in order to recover Dark Energy at low redshift, their expansion histories are obviously degenerate at late times. To break such a degeneracy, it is required an improvement on the knowledge of the early universe expansion curve: this aim can be achieved only by an accurate determination of the higher order parameters - and higher terms in the cosmographic expansion can be consistently reached only using very high redshift data.

The recent analysis Xia et al.(2012) handles the problem of interpreting the whole ensemble of cosmological data sets under a cosmographic perspective. We constrain the cosmographic parameters appearing in the expansions of the characteristic scales associated to Supernovae Type Ia (SNeIa), Gamma Ray Bursts (GRBs), Baryon Acoustic Oscillations (BAOs), the Cosmic Microwave Background (CMB) power spectrum and the determination of the Hubble parameter, estimated from surveys of galaxies (Hub).

Depending on the quantity that has been measured, it could be more appropriate to consider a particular cosmological distant scale than another one. To different distant scales correspond different Taylor expansions whose coefficients will combine the cosmographic parameters in different ways. In our analysis we refer to luminosity distance as the most direct choice for SNeIa and GRBs; volume distance for BAOs; angular diameter distance for the CMB. The inclusion of the direct estimation of the Hubble parameter is pretty peculiar and will be discussed later.

Even though the prominent role of SNe (in the high- $z$ version too) in doing the job is well-known, the potentiality of GRBs as cosmological standard candles has been recently explored as a possible proposal to increase the number of high redshift distance ladders. Data coming from the observations of both SNe and GRBs are used to fit directly the expression for the luminosity distance. The analysis is performed by using Monte Carlo Markov Chains in the multidimensional parameter space to derive the likelihood.

We have also used the BAO (albeit the cosmographic series fitting is only mildly improved): although the physics and the data of BAOs depend on the content in matter of the universe, the impact of spacetime priors on the power spectrum and the volume distances ratio were shown to be only weakly dependent on dynamical feature, leaving the safe possibility to use them as a further constraining tool.

The CMB data account for a very stable and well determined scale. It is worth noting here, anyway, that on the contrary of the other probes, CMB data provide the problem of a lack of universality in the cosmographic approach. Unfortunately, the set of parameters extracts from CMB observations is not truly independent from the dynamics of the underlying gravitational theory. Its definition, in fact, strictly depends on the assumption of a cosmological model that behaves as General Relativity plus a content of matter of arbitrary nature. It is hence impossible to use it straightforwardly within a purely cosmographic analysis which wants to apply also to non-standard cosmologies (based on exotic modified gravity theories). In Xia et al.(2012) we proposed CMB data constraints on the cosmographic series by restricting the results to a slightly smaller variety of models (namely models having standard physics up to the decoupling era, and whose eventual new physics after decoupling only modifies the small angle spectrum changing the overall amplitude and the angular diameter distance at the decoupling). A desirable full solution to this problem would be achieved "standardizing" somehow the CMB parameters or alternatively identifying other CMB observables which could be used as standard rulers.

Last probe is the direct determination of the Hubble parameter as determined by the differential ages of galaxies, with a caveat: the coefficient of the $n$-th $y$-power in the Taylor expansion already provides a combination of $n$ cosmographic parameters, while the same number of parameters appears only at the $(n+1)$-th power of the series expansion for the other distant scales. This is due to an extra derivative with respect to time included in the definition of the Hubble parameter. For this reason, and for the different nature of 
the Hubble data, we initially consider constraints based on standard candles and rulers, and then we add the Hubble data using one order less in the $y$-power expansion.

\section{Statistics selection between two truncations}

Higher order powers of the redshift expansions always improve the data fitting, since more free parameters are involved. However, for a given data set, there will be an upper bound on the order which is statistically significant in the data analysis (Vitagliano et al.(2010)). An early truncation of the power series also leads to several inconsistencies or artifacts. This justifies the search of some criteria to make a proper choice between two alternative models. The criterion that we use is the so-called F-test. This test compares two nested models (in this case, two different truncations of the Taylor series) in order to find out which is, for a given data set, the most viable approximation of the series. Supposing that the null hypothesis implies the correctness of the first model, the F-test verifies the probability for the alternative model to fit the data as well. If this probability is high, then no statistical benefit comes from the extra degrees of freedom associated to the new model. The less is this probability, the better is the data fitting of the second model against the first one.

The following Table summarizes the estimates obtained in our analysis for the most statistically meaningful (in the F-test sense) term of the series expansion.

\begin{tabular}{|c|c|c|c|c|c|}
\hline Data & \multicolumn{5}{|c|}{$\mathrm{SNIa}+\mathrm{GRB}+\mathrm{BAO}+\mathrm{CMB}\left(5^{t h}\right.$ order $)$} \\
\hline Parameter| & $q_{0}$ & $j_{0}$ & $s_{0}$ & $c_{0}$ & $H_{0}$ \\
\hline $\begin{array}{l}\text { Best Fit } \\
\text { Mean }\end{array}$ & $\begin{array}{c}-0.17 \\
-0.49 \pm 0.29\end{array}$ & $\begin{array}{c}-6.92 \\
-0.50 \pm 4.74\end{array}$ & $\begin{array}{c}-74.18 \\
-9.31 \pm 42.96\end{array}$ & $\begin{array}{c}-10.58 \\
126.67 \pm 190.15\end{array}$ & - \\
\hline$\chi_{\min }^{2} /$ d.o.f. & \multicolumn{5}{|c|}{$627.61 / 624$} \\
\hline Data & \multicolumn{5}{|c|}{$\mathrm{SNIa}+\mathrm{GRB}+\mathrm{BAO}+\mathrm{CMB}\left(5^{t h}\right.$ order $)+\mathrm{Hub}\left(4^{t h}\right.$ order $)$} \\
\hline Parameter| & $q_{0}$ & $j_{0}$ & $s_{0}$ & $c_{0}$ & $H_{0}$ \\
\hline $\begin{array}{l}\text { Best Fit } \\
\text { Mean }\end{array}$ & $\begin{array}{c}-0.24 \\
-0.30 \pm 0.16\end{array}$ & $\begin{array}{c}-4.82 \\
-4.62 \pm 1.74\end{array}$ & $\begin{array}{c}-47.87 \\
-41.05 \pm 20.90\end{array}$ & $\begin{array}{c}-49.08 \\
-3.50 \pm 105.37\end{array}$ & $\begin{array}{c}71.65 \\
71.16 \pm 3.08\end{array}$ \\
\hline$\chi_{\min }^{2} /$ d.o.f. & \multicolumn{5}{|c|}{$639.81 / 633$} \\
\hline
\end{tabular}

Comparing the parameters against the guess for different cosmological models (see Xia et al.(2012) for details), it is interesting to stress the remarkably good performance of $\Lambda \mathrm{CDM}$, even with respect to a cosmographic expansion with more free parameters. This could be taken as a strong hint in favor of this specific solution. However, we should warn about the (ab)use of a statistical comparison in terms of the derived $\chi^{2}$. In fact, while this procedure is completely meaningful for a selection between two nested cosmographic expansions, it becomes rather questionable when the comparison is between any fiducial cosmological model and a cosmographic series.

VV is supported by FCT - Portugal through the grant SFRH/BPD/77678/2011.

\section{References}

C. Cattoen \& M. Visser, 2007 Class. Quant. Grav. 24, 5985.

M. Visser, 2004 Class. Quant. Grav. 21, 2603.

V. Vitagliano, J. -Q. Xia, S. Liberati \& M. Viel, 2010 JCAP 1003, 005.

J. -Q. Xia, V. Vitagliano, S. Liberati \& M. Viel, 2012 Phys. Rev. D 85, 043520. 